\title{
Studies on Chitin XII. Chitin Derivatives Having 1,4-Dihydronicotinamide Groups for Asymmetric Reduction
}

\author{
Keisuke KURITA, Yoshiyuki KoYAMA, Kaori MURAKAMI, \\ Seiji YosHIDA, and Nguyen CHAU \\ Department of Industrial Chemistry, Faculty of Engineering, \\ Seikei University, Musashino-shi, Tokyo 180, Japan
}

(Received January 16, 1986)

\begin{abstract}
Chitin derivatives having 1,4-dihydronicotinamide groups (NADH model groups) were prepared and evaluated as polymeric asymmetric reducing agents. Water-soluble chitin and chitosan were efficiently nicotinoylated with nicotinic anhydride in aq. pyridine or aq. acetic acid/methanol under almost homogeneous highly swollen or homogeneous conditions. The subsequent quaternization and reduction of the pendant nicotinamide groups gave dihydronicotinamide groups immobilized on chitins. These derivatives were found to be effective for the asymmetric reduction of ethyl benzoylformate to ethyl mandelate with fairly high optical purity.

KEY WORDS Chitin / Water-Soluble Chitin / Chitosan / Polymeric Support / Immobilization / Dihydronicotinamide / Nicotinamide Adenine Dinucleotide / Asymmetric Reduction /
\end{abstract}

Polysaccharides have attracted much attention recently as polymeric supports in various fields such as enzyme immobilization and chromatography. In particular, partially deacetylated chitin and chitosan seem to have high potentials on account of their free amino groups and easy accessibility; we have thus far explored effective methods of chitin modification under homogeneous or almost homogeneous conditions using a $50 \%$ deacetylated sample (water-soluble chitin). ${ }^{1-3}$

Our interest in the utilization of chitin and its derivatives as polymeric supports prompted us to examine the possibility of the immobilization of NADH (a reduced form of nicotinamide adenine dinucleotide) models on chitin to form polymeric reducing agents capable of asymmetric reduction. NADH models have been reported to be incorporated into several kinds of synthetic polymers. ${ }^{4-10}$ These derivatives reduce ketones to alcohols, though the optical yield is not high.

In our attempt to immobilize NADH model compounds, water-soluble chitin with $50 \%$ deacetylation and chitosan with $95 \%$ deacetylation were chosen as supports to enable the efficient substitution reaction under homogeneous or almost homogeneous conditions. In addition to the facility of controlled reactions on these deacetylated chitins owing to the presence of amino groups and possible homogeneous reaction conditions the chiral fields offered by the polysaccharide backbones are anticipated to 'favorably influence the stereochemistry of the reduction thereby to improve optical yield. The active 1,4-dihydronicotinamide groups were, in some cases, made apart from backbones with spacers for increased accessibility. ${ }^{9,10}$ However, to take advantage of the chiral influence of chitin molecules fully, the groups should be linked to the polysaccharide chains as closely as possible, although 
the accessibility of the groups and thus the reduction yield may be sacrificed to some extent. This paper describes the immobilization of an NADH model on water-soluble chitin and chitosan by direct linking of dihydronicotinoyl groups to the amino groups of these polysaccharides and also the asymmetric reduction by the resulting polymeric reducing agents.

\section{EXPERIMENTAL}

\section{Materials}

Chitin was isolated from shrimp shells. Chitosan was prepared by treating chitin with $50 \%$ aqueous sodium hydroxide at 135 ${ }^{\circ} \mathrm{C}$ for $3.5 \mathrm{~h}$. The degree of deacetylation was $95 \%$ as determined by IR spectroscopy. ${ }^{11}$ Water-soluble chitin with $50 \%$ deacetylation was prepared according to the procedure reported previously. ${ }^{12}$ Nicotinic anhydride was synthesized from nicotinoyl chloride and potassium nicotinate; mp $122-124^{\circ} \mathrm{C}$ (lit. $\left.{ }^{13} 122-123^{\circ} \mathrm{C}\right)$.

\section{Reaction of Water-Soluble Chitin and chitosan with Nicotinic Anhydride}

(1) In Pyridine: A solution of $0.50 \mathrm{~g}$ of watersoluble chitin in $35 \mathrm{ml}$ of water was poured into $300 \mathrm{ml}$ of pyridine to give a highly swollen precipitate. To this mixture was added a solution of $3.1 \mathrm{~g}$ (10-fold excess on the basis of amino groups of water-soluble chitin) of nicotinic anhydride in $35 \mathrm{ml}$ of pyridine with stirring at room temperature. The reaction was discontinued after $24 \mathrm{~h}$ and the mixture was poured into acetone. The precipitate (Ia) was washed with acetone, dried, and then treated with $100 \mathrm{ml}$ of $5 \%$ aqueous sodium carbonate for two days at room temperature. The solid was filtered, washed with water to neutral, and dried to give $0.47 \mathrm{~g}$ of the product (IIa) as a white powder.

(2) In Acetic Acid/Methanol: To a solution of $0.50 \mathrm{~g}$ of water-soluble chitin in $25 \mathrm{ml}$ of $10 \%$ aqueous acetic acid were added $50 \mathrm{ml}$ of methanol and then $3.1 \mathrm{~g}$ (10-fold excess) of nicotinic anhydride with stirring. Additional methanol, $150 \mathrm{ml}$, was added in three portions at a few-minute intervals. After $24 \mathrm{~h}$ stirring, the resulting viscous gelatinous mixture was poured into $700 \mathrm{ml}$ of acetone to precipitate the polymer which was filtered and washed with acetone thoroughly. The dried product (Ib) was treated with $100 \mathrm{ml}$ of $5 \%$ aqueous sodium carbonate for 2 days. It was washed with water and dried in vacuo. The yield of the white powder (IIb) was $0.42 \mathrm{~g}$.

In a similar manner, $0.49 \mathrm{~g}$ of a chitosan derivative having nicotinamide groups (IIc) was obtained from $0.50 \mathrm{~g}$ of chitosan and $6.6 \mathrm{~g}$ (10-fold excess) of nicotinic anhydride.

\section{Quaternization of Nicotinamide Groups with Benzyl Chloride}

The powdered nicotinamide derivative (IIa), $0.47 \mathrm{~g}$, was added to a solution of $0.38 \mathrm{~g}$ (about 3-fold excess) of benzyl chloride in $20 \mathrm{ml}$ of dimethylformamide. The mixture was heated at $80^{\circ} \mathrm{C}$ for $48 \mathrm{~h}$ and then poured into methanol. The solid was filtered, washed with methanol, and dried to give $0.52 \mathrm{~g}$ of the product (IIIa) as a light yellow powdery material.

Similarly, nicotinamide derivatives IIb and IIc were quaternized to give IIIb and IIIc, respectively.

\section{Reduction of Benzylpyridinium Groups}

To a dispersion of $0.52 \mathrm{~g}$ of quaternized derivative IIIa in $50 \mathrm{ml}$ of methanol were added $0.5 \mathrm{~g}$ of potassium carbonate in $50 \mathrm{ml}$ of water and $0.5 \mathrm{~g}$ of sodium hydrosulfite in $50 \mathrm{ml}$ of water. After stirring the mixture at room temperature for $24 \mathrm{~h}$, the solid was filtered, washed with deionized water and methanol, and dried to give $0.50 \mathrm{~g}$ of a light yellow powder (IVa).

\section{Determination of Dihydronicotinamide Groups}

The amount of dihydronicotinamide groups 
immobilized on the polysaccharides was determined by oxidation-reduction titration. ${ }^{6}$ The polysaccharides having dihydronicotinamide groups were treated with aqueous potassium ferricyanide and the excess ferricyanide was titrated with aqueous sodium thiosulfate.

\section{Reduction of Ethyl Benzoylformate with Chitin Derivatives}

A dispersion of $0.178 \mathrm{~g}(1 \mathrm{mmol})$ of ethyl benzoylformate, $1.21 \mathrm{~g}$ of IVa containing 1.5 mmol of dihydronicotinamide groups, and $0.124 \mathrm{~g}(1 \mathrm{mmol})$ of magnesium perchlorate in $17 \mathrm{ml}$ of dry acetonitrile was heated at 40 ${ }^{\circ} \mathrm{C}$ for $72 \mathrm{~h}$. The solvent was removed and $15 \mathrm{ml}$ of water was added to the residue. The mixture was extracted with ether. After evaporation of the extract, $50 \mathrm{ml}$ of water was added and the mixture was extracted again with three $20 \mathrm{ml}$-portions of dichloromethane. Removal of the solvent gave $0.088 \mathrm{~g}$ of a residual oil. HPLC analysis (column; Jasco Finepak SIL $\mathrm{C}_{18}$, eluent: acetonitrile/ water $(7: 3)$ ) showed the content of ethyl mandelate and ethyl benzoylformate to be $13.3 \%$ and $80.8 \%$, respectively, which correspond to $0.012 \mathrm{~g}(6.5 \%$ yield on the basis of the starting ethyl benzoylformate) and $0.071 \mathrm{~g}$ $(40.0 \%$ recovery $)$.

\section{RESULTS AND DISCUSSION}

Incorporation of NADH groups into watersoluble chitin and chitosan was attained by a sequence of reactions involving first nicotinoylation of the amino groups, quaternization of the pyridyl nitrogen with benzyl chloride, and reduction to form 1,4-dihydronicotinamide groups as shown in Scheme 1.

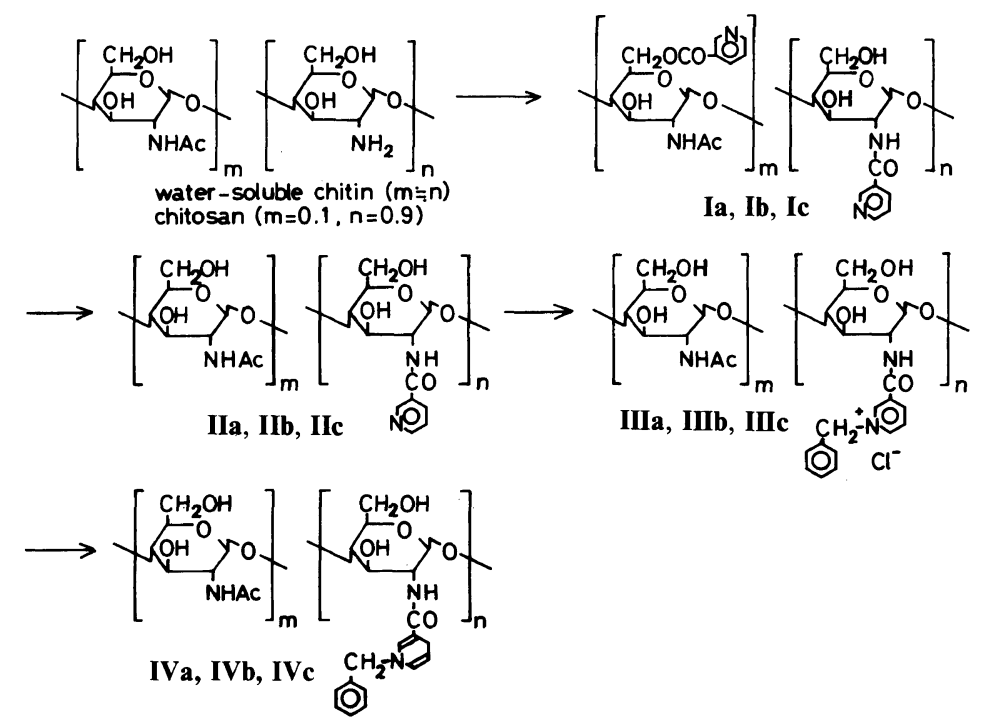

Scheme 1

Reaction of Water-Soluble Chitin and Chitosan with Nicotinic Anhydride

Partially deacetylated chitins prepared by homogeneous hydrolysis show high hydrophilicity, and a sample with about $50 \%$ deacetylation is water-soluble. This water-soluble chitin was treated with excess nicotinic anhydride in aqueous pyridine or in aqueous acetic acid/methanol.

Water-soluble chitin forms a highly swollen precipitate by precipitation from water to pyridine or dimethylformamide (DMF) and modification reactions can be efficiently car- 
ried out under almost homogeneous conditions. ${ }^{1-3}$ A swollen precipitate of water-soluble chitin in aqueous pyridine was thus treated with nicotinic anhydride in a manner similar to that employed in the previous acylation studies. ${ }^{1,2}$ The nicotinoylation proceeded smoothly at room temperature to form Ia.

Aqueous acetic acid is a good solvent for water-soluble chitin to give a clear solution, and nicotinoylation was also attempted in this solvent. Methanol was effective as the cosolvent to prevent precipitation of the acid anhydride and product, and coagulation of the product in isolation procedures. For $0.5 \mathrm{~g}$ of water-soluble chitin, $25 \mathrm{ml}$ of $10 \%$ aqueous acetic acid and about $200 \mathrm{ml}$ of methanol appeared appropriate for smooth reactions to give Ib. The state of the reaction mixture was, however, influenced by the mode of methanol addition. When the full amount of methanol was added at the beginning, the mixture became a rather hard gel, losing its fluidity. The methanol should be added in four portions at a few-minute intervals to keep the mixture fluid, which would be essential for homogeneous substitution.

Chitosan is also soluble in aqueous acetic acid, and the solution can be diluted with methanol as in the case of water-soluble chitin. Nicotinoylation of chitosan was performed similarly in the mixed solvent system, giving rise to the formation of Ic. Methanol was also added portionwise to prevent the formation of hard gels.

In all the IR spectra of nicotinoylated products from both water-soluble chitin and chitosan isolated by pouring the reaction mixtures into acetone, an absorption at around $1730 \mathrm{~cm}^{-1}$ was observed along with strong ones at 1650 and $1550 \mathrm{~cm}^{-1}$, indicating that the substitution at hydroxyl groups occurred to some extent besides at amino groups. The ester band was only a shoulder when the reaction was carried out in aqueous acetic acid/methanol, but became a distinct medi- um absorption in aqueous pyridine. This reveals that the acid anhydride is a much more powerful, thus less selective, reagent under highly swollen state in aqueous pyridine than under homogeneous conditions in aqueous acetic acid/methanol.

In subsequent reactions, especially in the final reaction with ethyl benzoylformate, the sample derived from the one having an ester absorption resulted in the formation of a complexed product composed of several components difficult to resolve. This complexity was probably brought about by the presence of ester linkages which are considered to cause some side reactions as a result of possible hydrolysis. Thus the nicotinoyl groups bonded to hydroxyl groups had to be removed at this stage. Alkaline hydrolytic cleavage of ester linkages was followed by IR spectroscopy using saturated aqueous sodium hydrogencarbonate. The ester band remained even after a week of treatment. Cleavage in aqueous sodium carbonate proceeded much more rapidly. The ester band was completely removed in a day in $10 \%$ sodium carbonate, but the other bands in IR spectra deformed a little, suggesting some side reactions to have occurred. The hydrolysis of ester linkages was complete in two days in $5 \%$ sodium carbonate, but the peaks began to deform slightly after three or four days. All the nicotinoylated products were thus treated with $5 \%$ sodium carbonate for two days to obtain selectively $\mathrm{N}$-nicotinoylated products (IIa, IIb, and IIc). Figure 1 illustrates the IR spectra of water-soluble chitin and nicotinoylated products before and after alkaline treatment (Ib and IIb). In the spectra of substituted derivatives, amide bands I and II became strong compared to those of watersoluble chitin. The products were obtained as almost colorless solids and the results are summarized in Table I. 


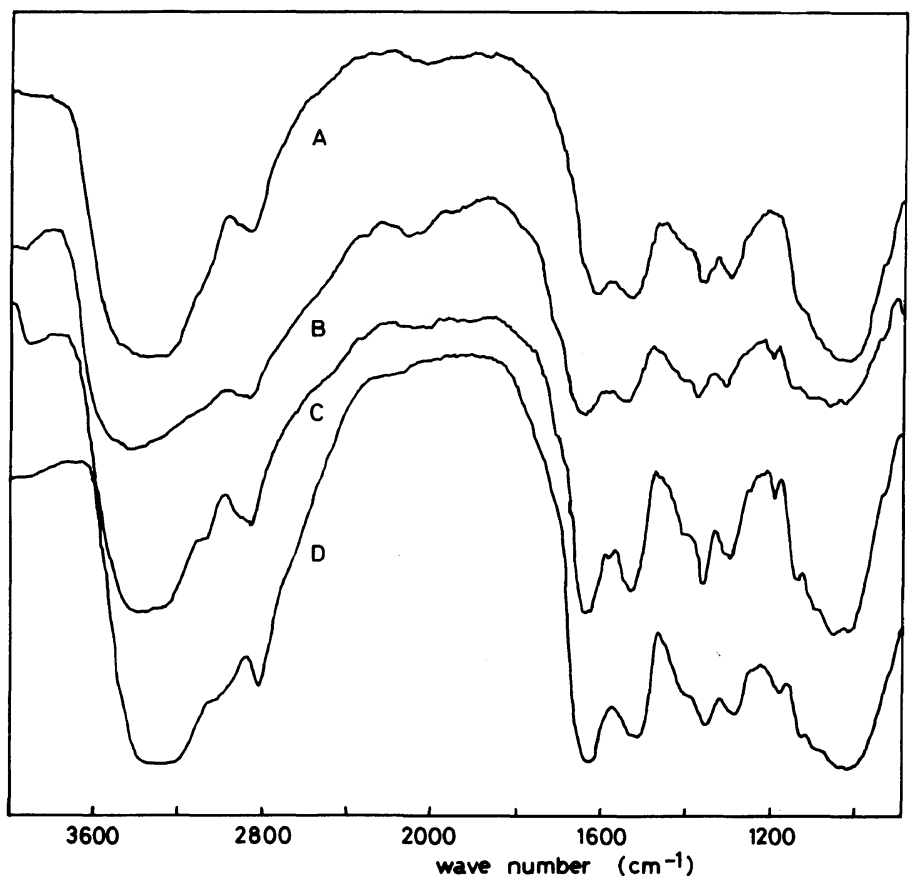

Figure 1. IR spectra (KBr) of water-soluble chitin derivatives: (A), water-soluble chitin; (B), Ib; (C), IIb; (D), IVb.

Table I. N-Nicotinoylation of water-soluble chitin and chitosan

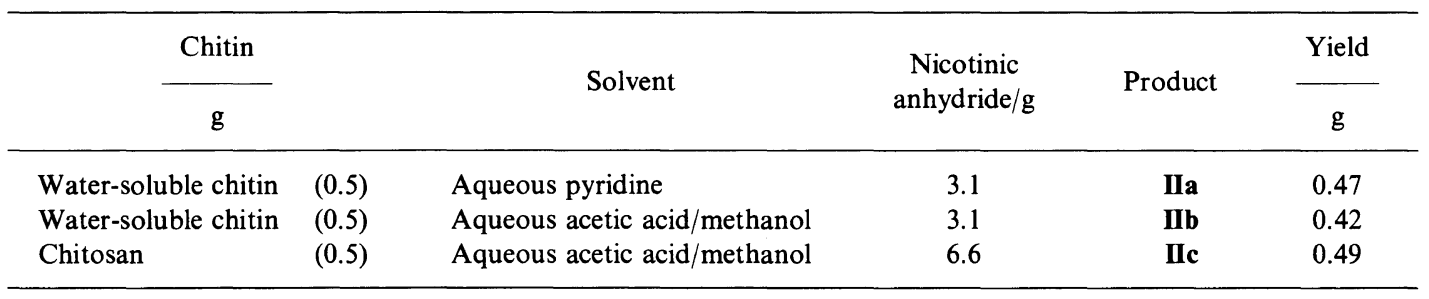

Quaternization and Reduction to Dihydronicotinamide Derivatives

The nicotinoylated products (IIa, IIb, and IIc) prepared above were then subjected to quaternization with about 3-fold excess benzyl chloride in DMF. The quaternized derivatives (IIIa, IIIb, and IIIc) were isolated as pale yellow powdery materials. The results are listed in Table II.

The nicotinamide groups were then reduced with sodium hydrosulfite and potassium carbonate in aqueous methanol. The resulting products with 1,4-dihydronicotinamide groups (IVa, IVb, and IVc) were obtained as pale yellow solids. The substitution degree of the dihydronicotinamide group was determined by the reported method of oxidationreduction titration using potassium ferricyanide as the oxidizing agent for the dihydronicotinamide group. The yield and substitution degree per pyranose unit are summarized in Table III. As shown in the table, about $15 \%$ and $30 \%$ of the pyranose rings had a dihydronicotinamide group in water-soluble chitin and chitosan derivatives, respectively. These values are, however, apparent substitution 
Table II. Quaternization of chitin derivatives having pendant nicotinamide groups

\begin{tabular}{cccc}
\hline Chitin derivative/g & Product & Yield $/ g$ \\
\hline IIa $(0.47)$ & IIIa & 0.52 \\
IIb $(0.49)$ & IIIb & 0.50 \\
IIc $(0.50)$ & IIIc & 0.58 \\
\hline
\end{tabular}

Table III. Reduction of nicotinamide groups to dihydronicotinamide groups in chitin derivatives

\begin{tabular}{ccccc}
\hline Chitin derivative/g & Product & Yield & $\begin{array}{c}\text { Degree of } \\
\text { Dubstitution }\end{array}$ \\
\cline { 4 - 5 } & & $\mathrm{g}$ & \\
\hline IIIa & $(0.52)$ & IVa & 0.50 & 0.17 \\
IIIb & $(0.50)$ & IVb & 0.48 & 0.14 \\
IIIc & $(0.58)$ & IVc & 0.48 & 0.27 \\
\hline
\end{tabular}

a The apparent number of dihydronicotinamide groups per pyranose ring as determined by oxidationreduction titration under heterogeneous conditions.

degrees, since the titration detected only the active groups available on the surface of the insoluble derivatives, and thus the real degrees of substitution by nicotinamide and dihydronicotinamide groups should be much higher. The IR spectra of these chitin derivatives were not very distinct from those of the derivatives before reduction. A typical example of the spectra is included in Figure 1.

\section{Asymmetric Reduction of Ethyl Benzoylformate with Chitin Derivatives Having Dihydro- nicotinamide Groups}

The reduction of ethyl benzoylformate was carried out with dihydronicotinamidecontaining chitin derivatives (IVa, IVb, and IVc) in acetonitrile in the presence of mag-

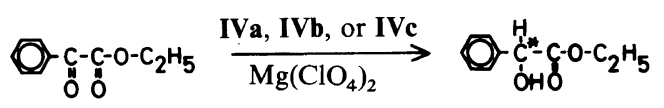

nesium perchlorate as suggested by Ohnishi and coworkers, ${ }^{14}$ and the extent of asymmetric reduction was elucidated by the optical rotation value of the product.
After the reduction, chitin derivatives could be removed easily by filtration and recovered in about $90 \%$ yields without appreciable change, judging from the IR spectra. The extraction residue obtained after the reaction was found to be a mixture of the expected ethyl mandelate and the starting ethyl benzoylformate. The composition was determined by HPLC and the results are summarized in Table IV. The yield of ethyl mandelate was generally not high $(6.5-8 \%)$ regardless of the kind of dihydronicotinamide derivative, i.e., IVa, IVb, or IVc, when these derivatives were used in about 1.5-fold excess on the basis of the molar ratio of the dihydronicotinamide group to ethyl benzoylformate. The low yield may be ascribed in part to the limited accessibility of the dihydronicotinamide groups for ethyl benzoylformate on account of the closeness of the active centers to the backbones, heterogeneous reaction conditions and low swelling ability, and also to the loss of ethyl mandelate in the extraction process. When further excess dihydronicotinamide derivatives $(3 \sim 6$ fold) were used, the yield of ethyl mandelate was improved to about $14 \%$.

After being used for the reduction of ethyl benzoylformate, these immobilized agents can be regenerated by reducing with sodium hydrosulfite. The regenerated IVa was found to be as effective as the original one; the yield of ethyl mandelate was $6.1 \%$ as compared with $6.5 \%$ by the original IVa under the same conditions.

Specific optical rotation of the resulting ethyl mandelate was then determined in ethanol in order to evaluate the dihydronicotinamide derivatives as polymeric asymmetricreducing agents. As shown in Table IV, the specific rotation values varied widely depending on the dihydronicotinamide derivatives. The values were especially high when watersoluble chitin derivatives (IVa and IVb) were used compared to that obtained with the chitosan derivative (IVc). These optical yields are 
Table IV. Asymmetric reduction of ethyl benzoylformate with chitin derivatives having dihydronicotinamide groups

\begin{tabular}{|c|c|c|c|c|c|}
\hline \multirow{2}{*}{$\begin{array}{l}\text { Chitin } \\
\text { derivative }\end{array}$} & Dihydronicotinamide group/mol & \multirow{2}{*}{$\begin{array}{l}\text { Recovery of ethyl } \\
\text { benzoylformate } / \%\end{array}$} & \multirow{2}{*}{$\begin{array}{l}\text { Yield of ethyl } \\
\text { mandelate } / \%\end{array}$} & \multirow{2}{*}{$\frac{[\alpha]_{\mathrm{D}}^{25 \mathrm{a}}}{\text { degree }}$} & \multirow{2}{*}{$\frac{\mathrm{OP}^{\mathrm{b}}}{\frac{\mathrm{o}}{\mathrm{o}}}$} \\
\hline & Ethyl benzoylformate/mol & & & & \\
\hline IVa & 1.5 & 40.0 & 6.5 & -34.59 & 33.3 \\
\hline IVa & 3.3 & 36.8 & 14.0 & - & \\
\hline IVb & 1.3 & 69.3 & 8.0 & -22.44 & 21.6 \\
\hline IVc & 1.5 & 76.9 & 7.0 & -3.70 & 3.6 \\
\hline IVc & 6 & 44.3 & 14.2 & - & \\
\hline
\end{tabular}

a Determined in ethanol.

b Optical purity. Pure ethyl mandelate: $[\alpha]_{D}^{24}=-104^{\circ} .{ }^{15}$

much higher than those reported thus far with NADH models immobilized on other polymeric materials; the highest reported value is $7.3 \%{ }^{9}{ }^{9}$

The stereochemistry of asymmetric reduction should thus be associated with the chiral field formed by the polysaccharide backbones. The high efficiency in the asymmetric selectivity of the water-soluble chitin derivatives compared to the chitosan derivative seems to suggest the importance of the acetamide groups on pyranose rings for forming a chiral environment to accomodate the substrate. This may also be partly ascribable to low degrees of substitution which would make the influence of the chiral field by polysaccharide backbones more evident at the reaction center.

Consequently, water-soluble chitin and chitosan were confirmed to be useful as polymeric supports for the NADH model compound, and the former was especially prominent in that its dihydronicotinamide derivatives exhibited a high potential in asymmetric reduction of carbonyl compounds, although the reduction yield was not high.

Acknowledgment. We are indebted to $\mathrm{Mr}$.
H. Kawabe for his technical assistance.

\section{REFERENCES}

1. K. Kurita, T. Sannan, and Y. Iwakura, Makromol. Chem., 178, 2595 (1977).

2. K. Kurita, H. Ichikawa, S. Ishizeki, H. Fujisaki, and Y. Iwakura, Makromol. Chem., 183, 1161 (1982).

3. K. Kurita, Y. Koyama, and A. Taniguchi, J. Appl. Polym. Sci., 31, 1169 (1986).

4. M. Okawara, T. Sasaoka, and E. Imoto, Kogyo Kagaku Zasshi, 65, 1652 (1962).

5. A. S. Lindsey, S. E. Hunt, and H. G. Samill, Polymer, 7, 479 (1966).

6. Y. Kurusu, K. Nakajima, and M. Okawara, Kogyo Kagaku Zasshi, 71, 934 (1968).

7. E. Tsuchida, E. Hasegawa, and H. Ohno, J. Polym. Sci., Polym. Chem. Ed., 15, 417 (1977).

8. T. Endo and M. Okawara, J. Polym. Sci., Polym. Chem. Ed., 17, 3667 (1979).

9. S. Shinkai, H. Tsuji, T. Sone, and O. Manabe, J. Polym. Sci., Polym. Lett. Ed., 19, 17 (1981).

10. T. Endo, T. Takada, and M. Okawara, J. Polym. Sci., Polym. Chem. Ed., 21, 603 (1983).

11. T. Sannan, K. Kurita, K. Ogura, and Y. Iwakura, Polymer, 19, 458 (1978).

12. K. Kurita, T. Sannan, and Y. Iwakura, Makromol. Chem., 178, 3197 (1977).

13. H. Rinderknecht and M. Gutenstein, Org. Synth., Coll. Vol., 5, 822 (1973).

14. Y. Ohnishi, M. Kagami, and A. Ohno, J. Am. Chem. Soc., 97, 4766 (1975).

15. R. Roger, J. Chem. Soc., 2168 (1932). 